\title{
V- type proton ATP-ase to target cancer cells with the aid of Cyanobacterial metallothionein
}

\author{
Srinivasan sakthivel ${ }^{1}$ and Yogeshwaran sakthivel ${ }^{2}$ \\ ${ }^{1}$ iNano (Interdisciplinary Nano-Science Center), Department of Molecular Biology and Genetics, University of \\ Aarhus, Aarhus 8000C - DENMARK \\ ${ }^{2}$ Sri Manakula Vinayagar Medical College and Hospital, Pondicherry, INDIA
}

\begin{abstract}
ATP-ases are a group of enzymes that utilizes ATP hydrolysis, and the subsequent release of energy, to achieve a cellular function. The cellular functions involving ATP-ases are plentiful and diverse including initiation of DNA replication, DNA repair and remodeling, protein folding and chaperoning, protein degradation, intracellular transport, and ion transport. A large number of these enzymes represent attractive drug targets, and drugs targeting ATP-ases, such as proton pump inhibitors. Two families of molecular chaperones, heat shock protein 90 and heat shock protein 70 , possess N-terminal nucleotide binding domains (NBD) and require ATP-ase activity for their functions. NBD is charged and highly polar in nature and there is no crystal structure yet published. These two families of ATP-ases represent significant therapeutic targets for the treatment of cancer. The ATP-ase activity of Hsp90, in interaction to Cyanobacterial mettalothionein is essential for targeting cancer affected cells and can be used highly as a drug targeting molecule. Inhibition of ATP-ase activity at nucleotide binding site of the Hsp90 leads to prevent tumor growth. Till now, only two antibiotics (ansamycin and geldanamycin) were discovered for inhibiting ATP-ase enzymes. This article discuss about Vtype proton ATP-ases, interaction with Cyanobacterial mettalothionein for targeting cancer spoiled cells in Cancer treatment.
\end{abstract}

\section{INTRODUCTION}

ATP-ase is a class of enzymes that hydrolyzes ATP (Adeonsine Tri Phosphate) and decomposes them into ADP (Adenosine Di Phosphate). In other words, ATP-ases are a class of enzymes that catalyze the decomposition of adenosine triphosphate (ATP) into adenosine diphosphate (ADP) and a free phosphate ion ${ }^{1}$. The energy released during dephosphorylation, enzyme harnesses to drive other chemical reactions. The most important fact is that this process is widely used in all known forms of life. ${ }^{2}$

ATP-ases are membrane-bound transporters that couples ion movement through a membrane with the synthesis or hydrolysis of a nucleotides, usually ATPs. Different forms of membrane-associated ATP-ases have evolved over time to meet specific demands of cells ${ }^{3}$. These ATP-ases have been classified as F-, V-, A-, P- and E- ATPases based on their functional differences. F-ATPases (F1FO-ATPases) in mitochondria, chloroplasts and bacterial plasma membranes are the prime producers of ATP, using the proton gradient generated by oxidative phosphorylation (mitochondria) or photosynthesis (chloroplasts). VATPases (V1VO-ATPases) are primarily found in eukaryotic vacuoles, catalysing ATP hydrolysis to transport solutes and lower $\mathrm{pH}$ in organelles like proton pump of lysosome. A-ATPases (A1AO-ATPases) are found in Archaea and function like F-ATPases. P-ATPases (E1E2-ATPases) are found in bacteria, fungi and in eukaryotic plasma membranes and organelles, and function to transport a variety of different ions across membranes. E-ATPases are cell-surface enzymes that hydrolyse a range of NTPs, including extracellular ATP. Some such enzymes are integral membrane proteins (anchored within biological membranes) and move solutes across the membrane, typically against their concentration gradient and are called trans-membrane ${ }^{4}$.

\section{PROTON ATP-ASE}

\section{ATP phosphohydrolase / H+ Exporting ATP-ase}

Proton ATP-ase or H+ ATP-ase is found in plants and fungi. The proton ATP-ase is responsible for catalyzing the following reaction.

$\mathrm{ATP}+\mathrm{H} 2 \mathrm{O}+\mathrm{H}+\mathrm{in} \rightleftharpoons \mathrm{ADP}+$ phosphate $+\mathrm{H}+$ out

The 3 substrates of this enzyme are ATP, $\mathrm{H} 2 \mathrm{O}$ and $\mathrm{H}+$ where as its three products are ADP, phosphate and $\mathrm{H}+$. Proton ATP-asebelong to the family of hydrolases ${ }^{5}$, specifically those acting on acid anhydrides to catalyse transmembrane movement of substances. To be specific, the protein is a part of the P-Type ATP-ase family.

A proton-pumping ATP-ase is present in the plasma membrane of plant cells where it sustains transport-related 
functions. This enzyme is encoded by a family of genes that shows signs of both transcriptional and posttranscriptional regulation ${ }^{6}$. The regulation of pmal, one of the Nicotiana H+-ATP-ase genes, was characterized with the help of the [beta]-glucuronidase (gusA) reporter gene in transgenic plants. pmal is active in the root epidermis, the stem cortex, and guard cells. This activity depends on developmental and growth conditions ${ }^{7}$. For instance, pmal activity in guard cells was strongly enhanced when the plant material (young seedlings or mature leaves) was incubated in liquid growth medium ${ }^{8}$. pmal is also expressed in several tissues of the reproductive organs where active transport is thought to occur but where scarcely any ATP-ase activity has been identified, namely in the tapetum, the pollen, the transmitting tissue, and the ovules ${ }^{9}$. Several pma genes have a long 5[prime] untranslated region (leader sequence) containing an upstream open reading frame (ORF). Analysis of translational and transcriptional fusions with gusA in transgenic plants suggests that the pmal leader sequence might activate translation of the main open reading frame, even though the URF is translated by a large majority of the scanning ribosomes ${ }^{10}$. As confirmation, transient expression experiments showed that the pmal leader causes a fourfold post-transcriptional increase of main open reading frame expression ${ }^{11}$. Deletion of the URF by site-directed mutagenesis stimulated the main open reading frame translation 2.7 -fold in an in vitro translational assay. These results are consistent with a regulatory mechanism involving translation re-initiation. Altogether, they suggest a fine, multilevel regulation of H+-ATP-ase activity in the plant ${ }^{12} . \mathrm{H}+-$ exporting ATP-ase is also known as proton ATP-ase or more simply proton pump. Other names in common use include proton translocating ATP-ase, yeast plasma membrane H+-ATP-ase, yeast plasma membrane ATP-ase, and ATP phosphohydrolase ${ }^{13}$.

Three major structural families of ATP-ases have been identified and characterized ${ }^{14}$. First, Most of the ATPases for which structures have been described contain the classical mononucleotide binding motif known as the Walker motif ${ }^{15}$. In comparison, Hsp90 belongs to a smaller subset of GHKL ATP-ases whose binding site is characterized by a left handed $\beta$-R- $\beta$ (Bergerat) fold. The GHKL ATP-ases are named after key family members: gyrase B, Hsp, histidine kinase, and MutL ${ }^{16}$. Hsp70 belongs to a third subset of ATP-ases that contain actin fold ${ }^{17}$. In this group of ATP-ases, the nucleotide binds in a cleft formed at the interface of two domains with a loop containing conserved residues and two $\beta$-hairpins forming interactions with adenine and the phosphate groups, respectively ${ }^{18}$. The very nature of the ATP binding pocket is a clear reason why targeting the NBD of Hsp70 has proved particularly challenging so far ${ }^{19}$. Hsp70 and Hsc70 exhibit a high degree of structural identity (>99\%) in the NBD, and therefore, small molecule inhibitors targeted against the NBD of Hsp70 are likely to inhibit Hsc70 with equi-potency. The following discussion will focus on the binding site of Hsp70, but the points and ideas raised here are equally applicable and relevant to $\mathrm{Hsc}^{20}{ }^{20}$.

\section{CYANOBACTERIAL METALLOTHIONEIN}

Bacterial Strains and Culture Conditions:

Synechococcus sp. PCC 7942 shall be cultured in BG-11 for 2 weeks under constant flourescent light (50 micromole quanta $\left.\mathrm{m}^{-2} \mathrm{~s}^{-1}\right)$ first on a shaker $(140 \mathrm{rpm})$ and later with air bubbling at $27^{\circ} \mathrm{C}^{21}$. Prochlorothrix hollandica shall be grown in BG-11 with constant flourescent light (40 micromole quanta $\left.\mathrm{m}^{-2} \mathrm{~s}^{-1}\right)$ and air bubbling at $25^{\circ} \mathrm{C}$ for two weeks ${ }^{22}$.

\section{Restriction Enzymes:}

The following enzymes can be used in the restriction digestion analysis, HaeIII, Hinfl, MspI, RsaI, and Taq ${ }^{23}$.

Polymerase Chain Reaction:

Metallothionein (SmtA) locus amplification can be performed using oligonucleotides ${ }^{24}$.

Sequence Alignment of the Class II Metallothioneins:

PIMA sequence alignment can be performed by using the algorithms ${ }^{25}$.

\section{CONCLUSION}

Thus this review article describes keenly about the interaction between Cyanobacterial metallothionein and V-type proton ATP-ases, which throws up its ability as a drug targeting agent for targeting cancer affected cells in Cancer treatment.

\section{REFERENCES}

1. Benson JD, Chen YN, Cornell-Kenon SA 2006. Validating cancer drug targets. Nature, 441: 451-456.

2. Biamonte MA, Van de WR, Arndt JW, Scannevin RH, Perret D et al,2010. Heat shock protein 90: inhibitors in clinical trials. J. Med. Chem., 53 (1), 3-17. 
3. Calderwood SK, Khaleque MA, Sawyer DB, Ciocca DR 2006. Heat shock proteins in cancer: chaperones of tumorigenesis. Trends Biochem. Sci., 31: 164-172.

4. Chene P 2002. ATPases as drug targets: learning from their structure. Nat. Rev. Drug Discov., 1 (9), 665-673.

5. Chiosis G, Vilenchik M, Kim J, Solit D 2004. Hsp90: the vulnerable chaperone. Drug Discov., 9: 881888 .

6. Collins I, Workman P 2006. New approaches to molecular cancer therapeutics. Nat. Chem. Biol., 2: 689700.

7. Da' Rocha Dias S, Friedlos F, Light Y 2005. Activated B-RAF is an Hsp90 client protein that is targeted by the anticancer drug 17-allylamino- 17-demethoxygeldanamycin. Cancer Res., 65: 10686-10691.

8. Drysdale MJ, Brough PA, Massey A, Jensen MR, Schoepfer J 2006. Targeting Hsp90 for the treatment of cancer. Curr. Opin. Drug Discovery Dev., 9 (4), 483-495.

9. Gaspar N, Sharp SY, Pacey S, Jones D, Walton Met al, 2009. Acquired resistance to 17-allylamino-17demethoxygeldanamycin (tanespimycin) in glioblastoma cells. Cancer Res., 69 (5), 1966-1975.

10. Grbovic OM, Basso AD, Sawai A 2006. V600E B-Raf requires the Hsp90 chaperone for stability and is degraded in response to Hsp90 inhibitors. Proc. Natl. Acad. Sci., 103: 57-62.

11. Greenman C, Stephans P, Smith R 2007. Patterns of somatic mutation in human cancer genomes. Nature, 446: 153-158.

12. Kamal AL,Thao J, Sensintaffar2003. A high-affinity conformation of Hsp90 confers tumor selectivity on Hsp90 inhibitors. Nature, 425: 407-410.

13. Kim YS, Alarcon SV, Lee S, Lee MJ,Giaccone Get al.2009. Update on Hsp90 inhibitors in clinical trial. Curr. Top. Med. Chem., 9 (15), 1479-1492.

14. Mayer MP, Bukau B 2005. Hsp70 chaperones: cellular functions and molecular mechanism. Cell. Mol. Life Sci., 62 (6), 670-684.

15. McDonald EP, Workman,Jones K 2007. Inhibitors of the HSP90 molecular chaperone: Attacking the master regulator in cancer. Curr.Top. Med. Chem., 6: 1091-1107.

16. Mimnaugh EG,Xu W,Vos M, Yuan X, Isaacs JSet al.2004. Simultaneous Inhibition of hsp 90 and the proteasome promotes protein ubiquitination, causes endoplasmic reticulum-derived cytosolic vacuolization, and enhances antitumor activity. Mol. Cancer Ther., 3 (5), 551-566.

17. Neckers L. 2006. Using natural product inhibitors to validate HSP90 as a molecular target in cancer. Curr. Med. Chem., 6: 1163-1171.

18. Nylandsted J, Brand K., Jaattela M2000. Heat shock protein 70 is Required for the survival of cancer cells. Ann. N.Y. Acad. Sci., 926, 122-125.

19. Nylandsted J, Wick W,Hirt UA, Brand K, Rohde M et al, 2002. Eradication of glioblastoma, and breast and colon carcinoma xenografts by Hsp70 depletion. Cancer Res., 62 (24), 7139-7142.

20. O'Reilly KE, Rojo F, She QB 2006. mTOR inhibition induces upstream receptor tyrosine kinase signaling and activates Akt. Cancer Res., 66:1500-1508.

21. Pearl LH, Prodromou C 2006. Structure and mechanism of Hsp90 molecular chaperone machinery. Ann. Rev. Biochem., 75: 271-294.

22. Pick E, Kluger Y, Giltnane JM 2007. High HSP90 expression is associated with decreased survival in breast cancer. Cancer Res., 67: 2932-2937.

23. Ritossa F 1962. A new puffing pattern induced by temperature shock and DNP in Drosophila. Experentia, 19: 571-573.

24. Turner JS and Robinson NJ 1995, Cyanobacterial Metallothioneins: Biochemistry and Molecular Genetics. J. Industrial Microbiology. 14: 119-125.

25. Smith, R.F. and T.F. Smith, 1990, Automatic Generation of Primary Sequence Patterns from Sets of Related Protein Sequences. PNAS, 87: 118-122. 